\title{
A Human Sodium-Dependent Vitamin C Transporter 2 Isoform Acts as a Dominant-Negative Inhibitor of Ascorbic Acid Transport
}

\author{
Eugene A. Lutsenko, Juan M. Carcamo, and David W. Golde* \\ Program in Molecular Pharmacology and Chemistry, Department of Medicine, and Department of Clinical Laboratories, \\ Memorial Sloan-Kettering Cancer Center, New York, New York 10021
}

Received 11 July 2003/Returned for modification 18 September 2003/Accepted 16 January 2004

\begin{abstract}
Vitamin $\mathrm{C}$ is transported as ascorbic acid (AA) through the sodium-ascorbate cotransporters (SVCT1 and -2) and as dehydroascorbic acid (DHA) through the facilitative glucose transporters. All cells have glucose transporters and take up DHA that is trapped intracellularly by reduction and accumulated as AA. SVCT2 is widely expressed in cells and tissues at the mRNA level; however, only specialized cells directly transport AA. We undertook a molecular analysis of SVCT2 expression and discovered a transcript encoding a short form of human SVCT2 (hSVCT2-short) in which 345 bp is deleted without a frame shift. The deletion involves domains 5 and 6 and part of domain 4. cDNA encoding this isoform was isolated and expressed in 293T cells, where the protein was detected on the plasma membrane. Transport studies, however, revealed that hSVCT2-short gave rise to a nonfunctional transporter protein. hSVCT2-short arises by alternative splicing and encodes a protein that strongly inhibited the function of SVCT2 and, to a lesser extent, SVCT1 in a dominant-negative manner, probably by protein-protein interaction. The expression of hSVCT2-short varies among cells. PCR analysis of cDNA isolated from melanocytes capable of transporting AA revealed a predominance of the full-length isoform, while HL-60 cells, which express SVCT2 at the mRNA level and were incapable of transporting AA, showed a predominance of the short isoform. These findings suggest a mechanism of AA uptake regulation whereby an alternative SVCT2 gene product inhibits transport through the two known AA transporters.
\end{abstract}

Vitamin $\mathrm{C}$ is essential for human health. Most mammals produce vitamin $\mathrm{C}$ in the liver; however, humans and other primates are unable to synthesize ascorbic acid (AA) and must obtain it from the diet $(9,13)$. Vitamin $\mathrm{C}$ is transported into cells in the oxidized form, dehydroascorbic acid (DHA), via facilitative glucose transporters (GLUTs) $(19,24)$ and as AA in specialized cells by sodium-dependent AA transporters (23). Two isoforms of the sodium-dependent vitamin $\mathrm{C}$ transporters (SVCTs) have been molecularly characterized in rats and humans $(3,10,18,23,28,29)$. Kyte-Doolittle hydropathy analysis (7) of the human SVCT2 (hSVCT2) amino acid sequence predicts a topographical model of a transporter with 12 transmembrane domains with both the $\mathrm{N}$ and $\mathrm{C}$ termini intracellular. The N-terminal (102-amino-acid) and the C-terminal (81amino-acid) tails in the cytoplasm are long and hydrophilic. The extracellular loop between transmembrane domains 3 and 4 contains two potential sites for N-glycosylation (Asn-188 and Asn-196). The hSVCT1 transporter is highly homologous to hSVCT2 with the same predicted membrane topology. An obvious difference between hSVCT1 and hSVCT2 is the additional sequences of 12 and 44 amino acids present in the $\mathrm{N}$ terminus of hSVCT2 at positions 2 and 38, respectively (10). The two isoforms of hSVCT differ in tissue distribution, as determined by Northern blot analysis, with hSVCT2 widely expressed at the mRNA level compared to hSVCT1. For hSVCT2, a 7.5-kb transcript was detected in most tissues tested, with the notable exceptions of the lung and skeletal muscle $(18,29)$. Probing for hSVCT1 resulted in a strong signal

\footnotetext{
* Corresponding author. Mailing address: Memorial Sloan-Kettering Cancer Center, 1275 York Ave., New York, NY 10021. Phone: (212) 639-8483. Fax: (212) 772-8589. E-mail: d-golde@ski.mskcc.org.
}

at $2.4 \mathrm{~kb}$ in the kidney, liver, small intestine, colon, ovary, and prostate $(28,29)$.

A comparison of the kinetic constants suggests that hSVCT2 has $\sim 10$-fold higher affinity for AA than hSVCT1 $(3,23)$. Uptake of AA via both hSVCT1 and hSVCT2 is absolutely dependent on the presence of $\mathrm{Na}^{+}$, and the replacement of $\mathrm{Na}^{+}$with either $\mathrm{Li}^{+}$or choline results in $>95 \%$ reduction in AA influx (3). Recent studies show that AA transport in mice (and probably in rats) is essential for perinatal survival. The knockout mouse lacking the mouse ortholog (Slc23a1 [for solute carrier family 23 member 1]) of a rat AA transporter died immediately after birth due to unexplained respiratory failure and cerebral hemorrhage (20).

We cloned and expressed hSVCT2 from human fetal brain tissue and discovered a short isoform that does not function as a transporter but rather acts as a dominant-negative inhibitor of AA transport through protein-protein interaction. The short isoform is widely expressed and can act as a regulator of AA uptake.

\section{MATERIALS AND METHODS}

Cell lines. Human kidney 293T cells were cultured in Dulbecco's high-glucose medium containing $10 \%$ fetal bovine serum, $1 \%$ penicillin-streptomycin, $1 \%$ sodium pyruvate, and 1\% L-glutamine. The stable cell line 293T-hSVCT2 was grown under the same conditions in the presence of 10 to $1,000 \mu \mathrm{g}$ of zeocin (Invitrogen)/ml. The 293T cells were transfected by the Ca-phosphate method at $1.5 \times 10^{6}$ cells per 100 -mm-diameter plate (15). After overnight incubation with the transfection cocktail, cells were either selected with zeocin to create stable cell lines or used for membrane extractions or to study the behavior of the sodium-dependent transporters by monitoring the uptake of radioactively labeled AA. Human myeloid HL-60 cells and melanocytes were cultured in Iscove's modified Dulbecco's medium containing $10 \%$ fetal bovine serum, $1 \%$ penicillin-streptomycin, $1 \%$ sodium pyruvate, and $1 \%$ L-glutamine. The cells were maintained in an incubator with $5 \% \mathrm{CO}_{2}$ in air at $37^{\circ} \mathrm{C}$.

AA and DHA uptake. Transfected or control 293T cells were detached from plates with $0.05 \%$ trypsin, washed with phosphate-buffered saline (PBS), and 
A

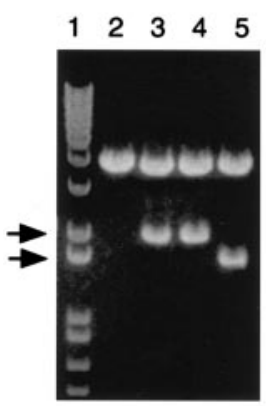

B

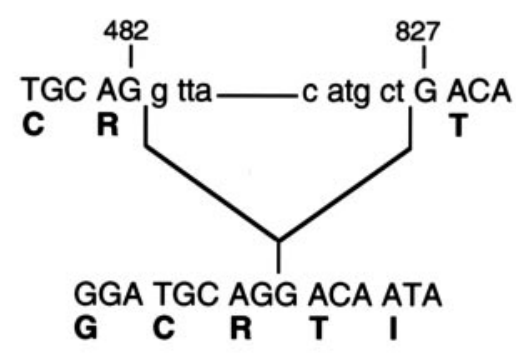

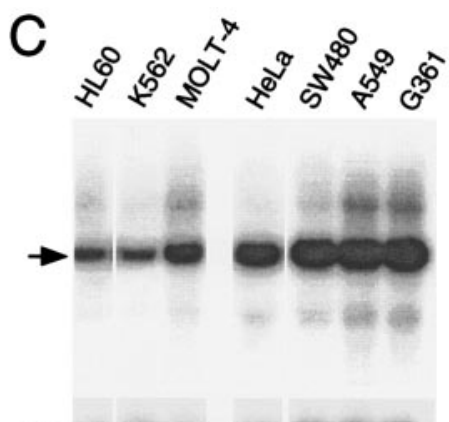

actin

FIG. 1. Cloning and distribution of full-length hSVCT2 and its short isoform. (A) Identification of full-length and short isoforms of hSVCT2 cDNA from fetal brain tissue was amplified using hSVCT2-3 and hSVCT2-5 primers (see Materials and Methods). The PCR products were separated in a $1 \%$ agarose gel, and bands at $2 \mathrm{~kb}$ were excised and cloned into the T/A vector. DNA isolated from the clones was digested with EcoRI. Lane 1, molecular weight marker; lane 2, empty T/A; lanes 3 and 4, T/A vector with full-length hSVCT2; lane 5, T/A vector with hSVCT2-short. Arrows, short and long isoforms. (B) Sequencing analysis of full-length hSVCT2 and its short isoform. (C) Northern blot analysis of hSVCT2 distribution in cell lines. A poly $\left(\mathrm{A}^{+}\right)$RNA master blot prepared from different cancer cell lines was probed with a DNA fragment obtained by BamHI digestion of full-length hSVCT2. Arrow, hSVCT2 RNA product.

held at room temperature for $20 \mathrm{~min}$ in incubation buffer (15 mM HEPES, 135 $\mathrm{mM} \mathrm{NaCl}, 1.8 \mathrm{mM} \mathrm{CaCl}_{2}, 0.8 \mathrm{mM} \mathrm{MgCl}_{2}$, and $5 \mathrm{mM} \mathrm{KCl}, \mathrm{pH}$ 7.4). Two million cells were added to incubation buffer with $100 \mu \mathrm{M}$ AA and $0.2 \mu \mathrm{Ci}$ of L-[1${ }^{14}$ C]AA (DuPont, Wilmington, Del.) for AA uptake studies or to a mixture containing AA and ascorbate oxidase for DHA uptake analysis (12). Following incubation, samples were washed twice with ice-cold calcium-magnesium-free PBS. After lysis in $10 \mathrm{mM}$ Tris- $\mathrm{HCl}(\mathrm{pH} 8.0)$ containing $0.2 \%$ sodium dodecyl sulfate (SDS), the incorporated radioactivity was determined by liquid scintillation. The amount of AA accumulated by $293 \mathrm{~T}$ cells was calculated using $1 \mu \mathrm{l} / 10^{6}$ cells as an estimate for the internal volume (12)

Membrane protein extractions and Western blot analysis. Cells were scraped into ice-cold HEPES buffer (10 mM HEPES, pH 7.4, $30 \mathrm{mM}$ mannitol, $10 \mathrm{mM}$ $\mathrm{CaCl}_{2}, 0.5 \mathrm{mM}$ PMSF, and protein inhibitors) (Roche, Indianapolis, Ind.). The cells were homogenized with a Dounce homogenizer, kept on ice for $10 \mathrm{~min}$, and centrifuged at 5,000 $\times g$ for $15 \mathrm{~min}$ at $4^{\circ} \mathrm{C}$, and the supernatant was collected and centrifuged for $30 \mathrm{~min}$ at $40,000 \times g$ and $4^{\circ} \mathrm{C}$. Plasma membrane-enriched pellets were prepared for SDS-polyacrylamide gel electrophoresis by incubation for $1 \mathrm{~h}$ at $37^{\circ} \mathrm{C}$ in the following loading buffer: $50 \mathrm{mM} \mathrm{Na}_{2} \mathrm{HPO}_{4}, \mathrm{pH} 6.8,4 \mathrm{M}$ urea, 0.25 $\mathrm{M}$ beta-mercaptoethnaol, $1 \%$ (wt/vol) SDS, and $0.0005 \%$ bromphenol blue. hSVCT2 was detected using anti-hSVCT2 antibody (Santa Cruz Biotechnology, Santa Cruz, Calif.).

Northern blot analysis. Multiple-cell-line RNA blots containing size-fractioned human poly $\left(\mathrm{A}^{+}\right)$RNA from cancer cell lines were obtained from Clontech (Palo Alto, Calif.). These blots were hybridized with a ${ }^{32} \mathrm{P}$-labeled fragment of hSVCT2 digested with BamHI corresponding to nucleotides 632 to 1449 Hybridization was carried out under highly stringent conditions at $68^{\circ} \mathrm{C}$ overnight with ExpressHyb hybridization solution (Stratagene, La Jolla, Calif.). The blots were washed three times at $50^{\circ} \mathrm{C}$ in $0.1 \times \mathrm{SSC}(1 \times \mathrm{SSC}$ is $0.15 \mathrm{M} \mathrm{NaCl}$ plus 0.015 $\mathrm{M}$ sodium citrate) containing $0.1 \%$ SDS and were exposed to XAR-5 film at $-70^{\circ} \mathrm{C}$ with two intensifying screens. The signals were visualized by autoradiography.

Cloning of hSVCT2 and its short isoform, hSVCT2-short. Human fetal brain cDNA (Clontech) was amplified using the Advantage-2 polymerase mix (Clontech) with the following primers: hSVCT2-5 (ATG ATG GGT ATT GGT AAG AAT ACC) and hSVCT2-3 (CTA TCC CGT GGC CTG GGA GTC TTC ATC). The PCR products were separated in $1 \%$ agarose gel, and fragments $\sim 2 \mathrm{~kb}$ long were purified from the gel and subcloned into the T/A cloning vector (Invitrogen). Clones were screened for hSVCT2, first by EcoRI digestion of isolated DNA and then by sequencing. Clones that had homology to hSVCT2 were subcloned into the EcoRI site of the mammalian expression vectors pcDNA4/ HisMaxC and pCMV-Tag4A (Invitrogen).

Subcloning hSVCT1. The open reading frame corresponding to hSVCT1 was amplified by Taq polymerase from pBlueScript SK $(-)$ (provided by the Matthias A. Hediger laboratory) using the following primers: hSVCT1-5 (ATG AGG GCC CAA GAG GAC CTC GAG G) and hSVCT1-3 (TCA GAC CTT GGT GCA CAC AGA TGC). The PCR products were separated on $1 \%$ agarose gel, and a fragment of $\sim 1,800 \mathrm{bp}$ was excised from a gel and cloned into $\mathrm{T} / \mathrm{A}$ cloning vector (Invitrogen). The clones were screened for hSVCT1, first by EcoRI digestion of isolated DNA and then by sequencing. Clones that had homology to hSVCT1 were subcloned into the EcoRI site of the mammalian expression vector pCMV-Tag2B (Invitrogen).

PCR detection of the short isoform of hSVCT2 in human tissues. PCR with Taq polymerase was performed on cDNAs from human fetal and adult tissues to detect the short isoform of hSVCT2 using the following primers: AAT2-300 (GGG GTC ACA GCA CTA CCT G) and AAT2-R900 (GGA TGG CCA GGA TGA TAG). The PCR products were separated on a $1 \%$ agarose gel and visualized by ethidium bromide staining. The anticipated length of the PCR product for the short isoform of hSVCT2 is $\sim 300 \mathrm{bp}$, whereas the full-length protein is $600 \mathrm{bp}$.

Immunostaining microscopy. Transfected $293 \mathrm{~T}$ cells were fixed with $4 \%$ paraformaldehyde for $20 \mathrm{~min}$ at room temperature. The cells were then permeabilized by adding $2 \%$ Triton $\mathrm{X}-100$ for $10 \mathrm{~min}$ at room temperature and incubated with anti-FLAG and/or anti-His epitope monoclonal antibody overnight at $4{ }^{\circ} \mathrm{C}$. After six washings with PBS, the cells were incubated for $1 \mathrm{~h}$ at room temperature with goat anti-mouse immunoglobulin $\mathrm{G}$ conjugated with fluorescein isothiocyanate and finally mixed with a drop of oil and mounted on a glass slide. The cells were visualized by fluorescence microscopy.

\section{RESULTS}

Cloning of full-length hSVCT2 and its short isoform. We cloned the full-length hSVCT2 and hSVCT2-short from a human fetal cDNA library using the primers described in Materials and Methods. The PCR products were cloned into the T/A cloning vector for initial analysis, and DNAs from the clones were isolated and digested with EcoRI restriction enzyme. Figure 1A shows the results of initial screening for fulllength hSVCT2. We identified two bands after restriction enzyme digestion: one corresponded to a full-length clone, while the other was smaller. We sequenced both clones and found full-length hSVCT2 and its short isoform. The short isoform is missing $345 \mathrm{bp}$, from nucleotides 482 to 827 , without a frame shift (Fig. 1B), corresponding to deletion of intracellular domains 5 and 6 and partial deletion of domain 4 . To study both transporters simultaneously, we subcloned them into mammalian expression vectors with different tags. The full-length transporter was subcloned into pCMV-Tag4A, which carries a FLAG tag at the $3^{\prime}$ end, and the short isoform was subcloned into pcDNA4/HisMaxC, which carries a His tag at the $5^{\prime}$ end. 

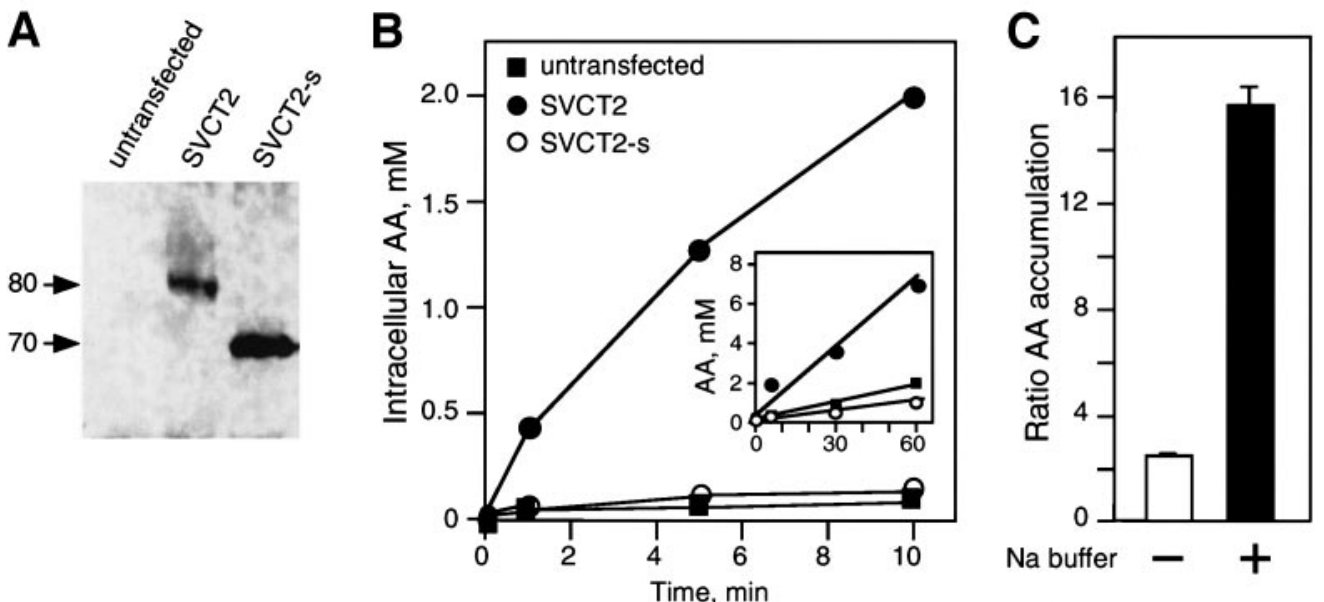

FIG. 2. Expression and functional characterization of hSVCT2 and its short isoform. (A) Western blot analysis of the expression of full-length hSVCT2 and its short isoform. 293T cells were transfected with $4 \mu \mathrm{g}$ of a plasmid carrying full-length hSVCT2 or its short isoform. The membrane was probed with commercially available antibodies against hSVCT2. Molecular masses are in kilodaltons. (B) Uptake studies in 293T cells. Cells were untransfected or transfected with full-length hSVCT2 (SVC2) or its short isoform (SVCT2-s). Uptake of radiolableled AA was performed at different time intervals. (Inset) Accumulation of ascorbic acid during longer incubations. (C) Sodium dependency of AA uptake mediated by hSVCT2. Cells were transfected with full-length hSVCT2, and uptakes were done with $(+)$ and without $(-)$ sodium in the buffer at 0 and 10 min. The ratios between accumulated AA at $10 \mathrm{~min}$ and that at $0 \mathrm{~min}$ are shown. The error bars indicate standard deviations.

Expression of hSVCT2 transporters at the mRNA level. To determine the general distribution of hSVCT2 transcript in human cells, we probed a commercially available RNA master blot containing poly $\left(\mathrm{A}^{+}\right)$RNAs from seven different cancer cell lines. The Northern blot analysis indicated that the hSVCT2-specific 7.5-kb transcript was present in all cell lines tested (Fig. 1C). The size of the hSVCT2-specific transcript was in agreement with previously published data $(18,29)$.

Expression of hSVCT2-short isoform in tissues. We investigated the distribution of the short isoform of hSVCT2 in different human tissues by PCR analysis. Commercially available human adult and fetal cDNA libraries were amplified using primers shown in Materials and Methods to detect the expression of the short isoform of hSVCT2 in these tissues. PCR products of $\sim 300 \mathrm{bp}$ (corresponding to the short isoform) were seen in all adult and fetal tissues tested (fetal brain, lung, liver, kidney, heart, muscle, thymus, and spleen and adult brain, lung, liver, kidney, heart, muscle, placenta, and pancreas). To be certain that these PCR products corresponded to the short isoform of hSVCT2, we sequenced two each (brain and lung) from adult and fetal tissue. The sequencing results revealed that both adult and fetal lung and brain tissues express the short isoform of hSVCT2.

Functional expression of hSVCT2 and its short isoform. To analyze the functionality of hSVCT2 and its short isoform, we cloned them into two mammalian expression vectors with a cytomegalovirus promoter, pCMV-Tag4A and pcDNA4HisMaxC, respectively. The full-length transporter carried the FLAG tag, and the short isoform carried the His tag. Plasmids encoding the transporters were separately transfected into the 293T cell line. The transfected cells were incubated for $24 \mathrm{~h}$ to allow the expression of the transporters on the plasma membrane. The membrane proteins were isolated from the transfected cells, separated by gel electrophoresis, and transferred onto nitrocellulose for probing with commercially available antibodies against hSVCT2 (Santa Cruz). Figure 2A shows that both transporters, full-length hSVCT2 and its short isoform, were expressed on the cell membrane. The apparent molecular masses of the full-length transporter and its short isoform were $\sim 80$ and $70 \mathrm{kDa}$, correlating with the sequencing results at the DNA level and indicating that the short isoform was missing 115 amino acids.

We expressed both transporters in 293T human kidney cells and measured the uptake of $\left[{ }^{14} \mathrm{C}\right]$ ascorbate at various time intervals. Cells transfected with the hSVCT2-short isoform were unable to transport AA. These cells behaved as an untransfected control, accumulating only $0.1 \mathrm{mM}$ intracellular AA after 10 min of incubation with labeled AA (Fig. 2B). On the other hand, cells transfected with full-length hSVCT2 accumulated $2 \mathrm{mM}$ AA intracellularly after $10 \mathrm{~min}$ of incubation (Fig. 2B). As expected, the inability of the short isoform to transport AA was also seen with longer incubation times. Cells transfected with the hSVCT2-short isoform behaved as an untransfected control, accumulating only $\sim 1 \mathrm{mM}$ intracellular AA after 60 min of incubation with labeled AA (Fig. 2B, insert). On the other hand, cells transfected with full-length hSVCT2 accumulated $7 \mathrm{mM}$ AA intracellularly after $60 \mathrm{~min}$ of incubation (Fig. 2B, insert). The transport of AA in the cells transfected with full-length hSVCT2 was sodium dependent. Cells transfected with the full-length transporter were unable to accumulate AA when the uptake studies were done in choline buffer lacking sodium. On the other hand, there was clear accumulation of AA when studies were conducted in incubation buffer supplemented with sodium (Fig. 2C).

The short isoform of hSVCT2 acts as a dominant-negative inhibitor of AA transport in 293T cells expressing full-length hSVCT2 via protein-protein interaction. To determine if expression of the short isoform of hSVCT2 had an effect on ascorbate transport in cells expressing full-length hSVCT2, we cotransfected 293 T cells with full-length hSVCT2 and increas- 

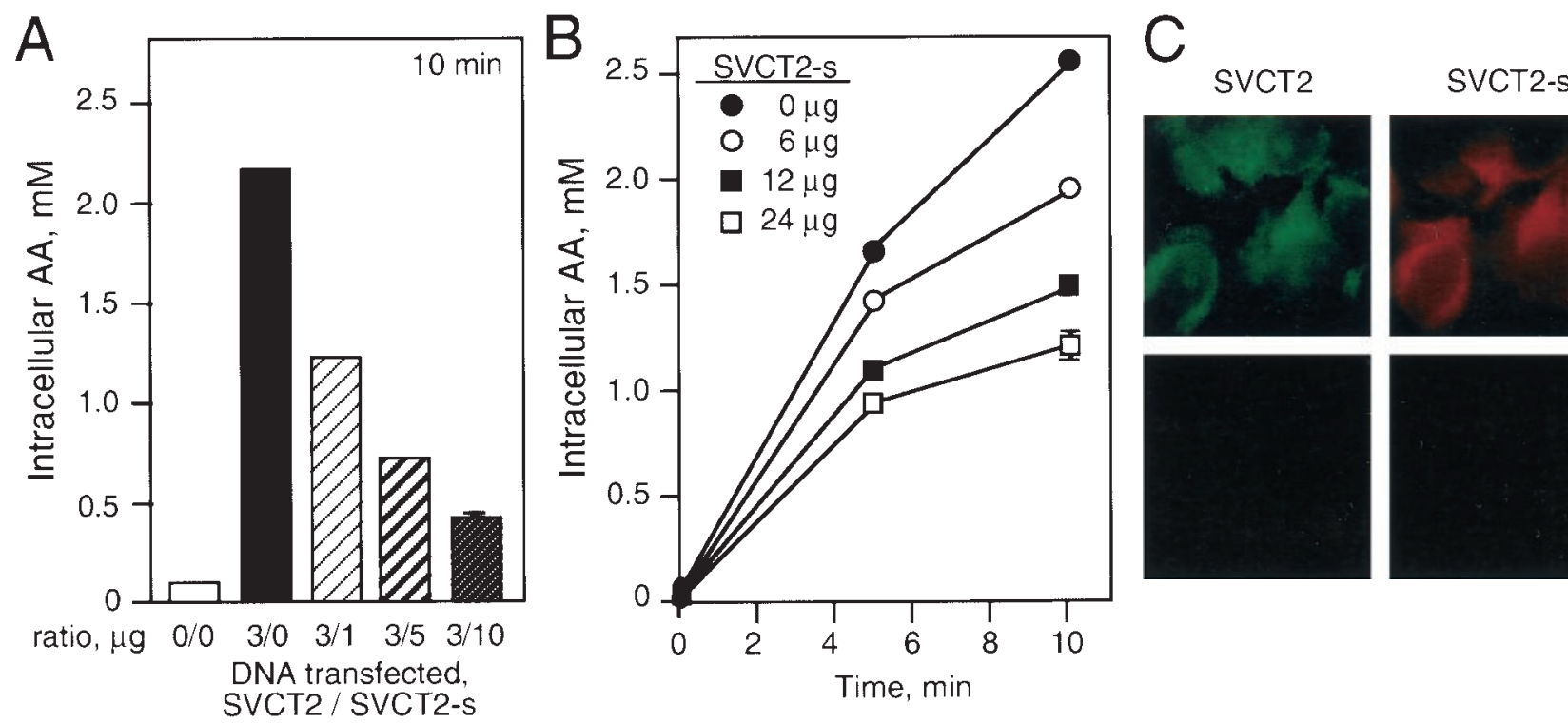

FIG. 3. Dominant-negative effect of hSVCT2-short isoform on AA uptake in 293T cells expressing full-length hSVCT2. (A) Cells were transfected with full-length hSVCT2 (SVCT2) or cotransfected with full-length hSVCT2 and increasing amounts of its short isoform (SVCT2-s). AA uptake was done at 0 and $10 \mathrm{~min}$. The error bar indicates the standard deviation. (B) Stable 293T cells expressing full-length hSVCT2 were transfected with increasing amounts of the hSVCT2-short isoform. AA uptake was measures at 0 , 5, and 10 min. (C) Cells were cotransfected with full-length hSVCT2 and its short isoform carrying FLAG and His tags, respectively. Immunostaining was performed the next day using either anti-His or anti-FLAG antibody. (Top) Cells cotransfected with full-length hSVCT2 and hSVCT2-short. (Bottom) Negative control experiment with nontransfected cells probed with either anti-His or anti-FLAG antibody.

ing amounts of its short isoform. Increasing amounts of the short isoform of hSVCT2 impaired ascorbate transport in 293T cells expressing a full-length transporter (Fig. 3A). Cells transfected with $3 \mu \mathrm{g}$ of full-length hSVCT2 and $10 \mu \mathrm{g}$ of the short isoform per 100 -mm-diameter plate accumulated only $0.5 \mathrm{mM}$ AA in $10 \mathrm{~min}$ compared to $2.2 \mathrm{mM}$ in the cells expressing only full-length hSVCT2. The reduction in intracellular accumulation of AA was found to be dose dependent with respect to hSVCT2-short. Impairment of AA transport in cells expressing full-length hSVCT2 was directly correlated with the amount of short isoform coexpressed in the cells (Fig. 3A). The inhibition of AA transport by hSVCT2-short could be measured when it was present in a ratio to full-length hSVCT2 as small as 1 to 3 (Fig. 3A).

We established a stable 293 T cell line expressing full-length hSVCT2 and transfected these cells with increasing amounts of hSVCT2-short to confirm the dominant-negative inhibitory effect on AA transport. Similar to the transiently transfected 293 T cells, we observed a reduction of intracellular AA accumulation when the stable cells were transfected with increasing amounts of the short isoform of hSVCT2 (Fig. 3B). Cells transfected with $24 \mu \mathrm{g}$ of hSVCT2-short accumulated $\sim 1 \mathrm{mM}$ AA intracellularly in $10 \mathrm{~min}$, whereas untransfected cells expressing only full-length hSVCT2 accumulated $2.5 \mathrm{mM}$ AA intracellularly after $10 \mathrm{~min}$ of incubation with labeled AA. The reduction in AA accumulation was correlated in a dose-dependent manner with the amount of the short isoform used for transfection of the cells (Fig. 3B).

To determine if both full-length hSVCT2 and its short isoform were coexpressed on the cell membrane, we performed immunostaining of the $293 \mathrm{~T}$ cells cotransfected with both wild- type hSVCT2 and its short isoform in a 1-to-1 ratio. These transporters carried distinctive tags in order to differentiate them during immunostaining. The full-length hSVCT2 had a FLAG tag, while its short isoform had a His tag. The results show that both transporters were coexpressed on the cell membrane (Fig. 3C). Controls using omission of primary or secondary antibodies revealed no labeling. The dominant-negative effect of hSVCT2-short acting when the full-length and the short isoforms are coexpressed at the cell surface suggests that the mechanism of inhibition is by protein-protein interaction.

The short isoform of hSVCT2 acts as a dominant-negative inhibitor of AA transport in 293T cells expressing full-length hSVCT1 via protein-protein interaction. Since hSVCT1 and hSVCT2 are highly homologous at the protein level (23), we reasoned that if the dominant-negative effect of hSVCT2-short is due to protein-protein complex formation, we could expect that a similar effect might be seen with cells expressing hSVCT1. Cells were cotransfected with full-length hSVCT1 and increasing amounts of the short isoform of hSVCT2. Increasing hSVCT2-short expression inhibited ascorbate uptake in $293 \mathrm{~T}$ cells expressing the full-length hSVCT1 transporter (Fig. 4A). Cells transfected with $1 \mu \mathrm{g}$ of full-length hSVCT1 accumulated $4.3 \mathrm{mM}$ AA intracellularly after 10 min of incubation with labeled AA, and this was decreased to $1.5 \mathrm{mM}$ in cells cotransfected with $15 \mu \mathrm{g}$ of short-isoform hSVCT2 per 100-mm-diameter plate (Fig. 4A). The reduction in AA uptake was correlated in a dose-dependent manner with the amount of hSVCT2-short isoform transfected (Fig. 4A). To see the inhibitory effect of hSVCT2-short on AA transport in cells expressing full-length hSVCT1 required an excess of the short isoform over hSVCT1. Significant inhibition of AA transport 
A

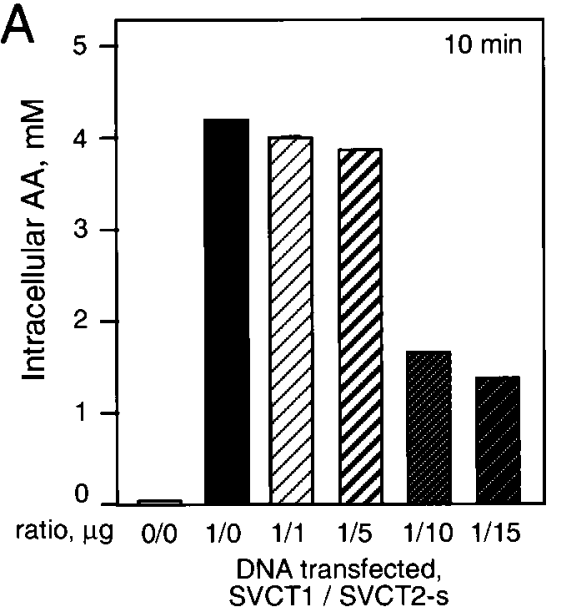

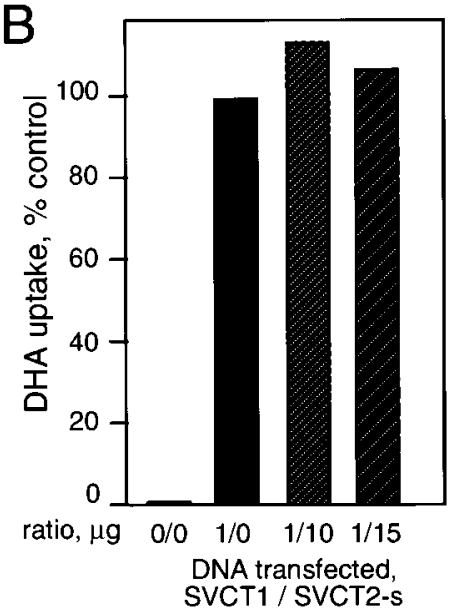

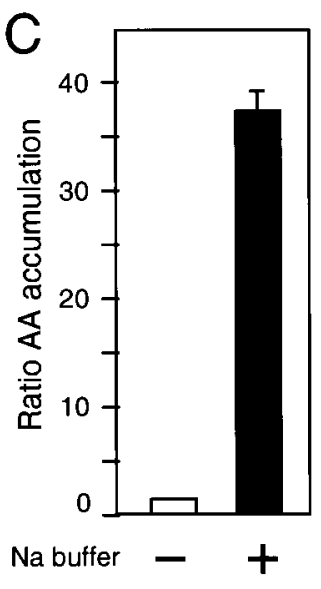

FIG. 4. Dominant-negative effect of hSVCT2-short isoform on AA uptake in 293T cells expressing full-length hSVCT1. (A) Cells were transfected with full-length hSVCT1 (SVCT1) or cotransfected with hSVCT1 and increasing amounts of the hSVCT2-short isoform (SVCT2-s). AA uptakes were measured at 0 and $10 \mathrm{~min}$. (B) Cells were cotransfected with hSVCT1 and increasing amounts of hSVCT2-short isoform. DHA uptakes were measures at 0 and $30 \mathrm{~min}$. Data are shown as percentages of control DHA uptake in cells transfected with hSVCT1. (C) Sodium dependency of AA uptake mediated by hSVCT1. Cells were transfected with full-length hSVCT1, and uptakes were done in the presence $(+)$ or absence (-) of sodium in the buffer. Ratios between accumulated AA at $10 \mathrm{~min}$ and that at 0 min are shown. The error bar indicates the standard deviation.

was seen, starting with a 10-to-1 ratio of the short isoform to hSVCT1 (Fig. 4A). Increasing amounts of the hSVCT2-short isoform had no functional effect on other membrane transporters, as evidenced by the ability of cells to take up DHA normally when cotransfected with full-length hSVCT2 and its short isoform (Fig. 4B). AA uptake by full-length hSVCT1 was sodium dependent, and cells transfected with hSVCT1 and incubated with choline buffer lacking sodium were unable to accumulate significant amounts of AA after 30 min of incubation with labeled AA. Cells incubated in the choline buffer supplemented with sodium showed a 32 -fold increase in accumulation of AA compared with the control (Fig. 4C).

Ability to transport $\mathrm{AA}$ is correlated in some cells with the amount of short isoform expressed. While all cells can transport vitamin C as DHA via the facilitative GLUTs, only specialized cells have the ability to transport AA directly in the form of ascorbate via sodium-dependent AA cotransporters. This is so even though hSVCT2 is widely expressed at the mRNA level $(18,29)$. We investigated this phenomenon in HL-60 cells that do not transport AA (25) (Fig. 5B) and in
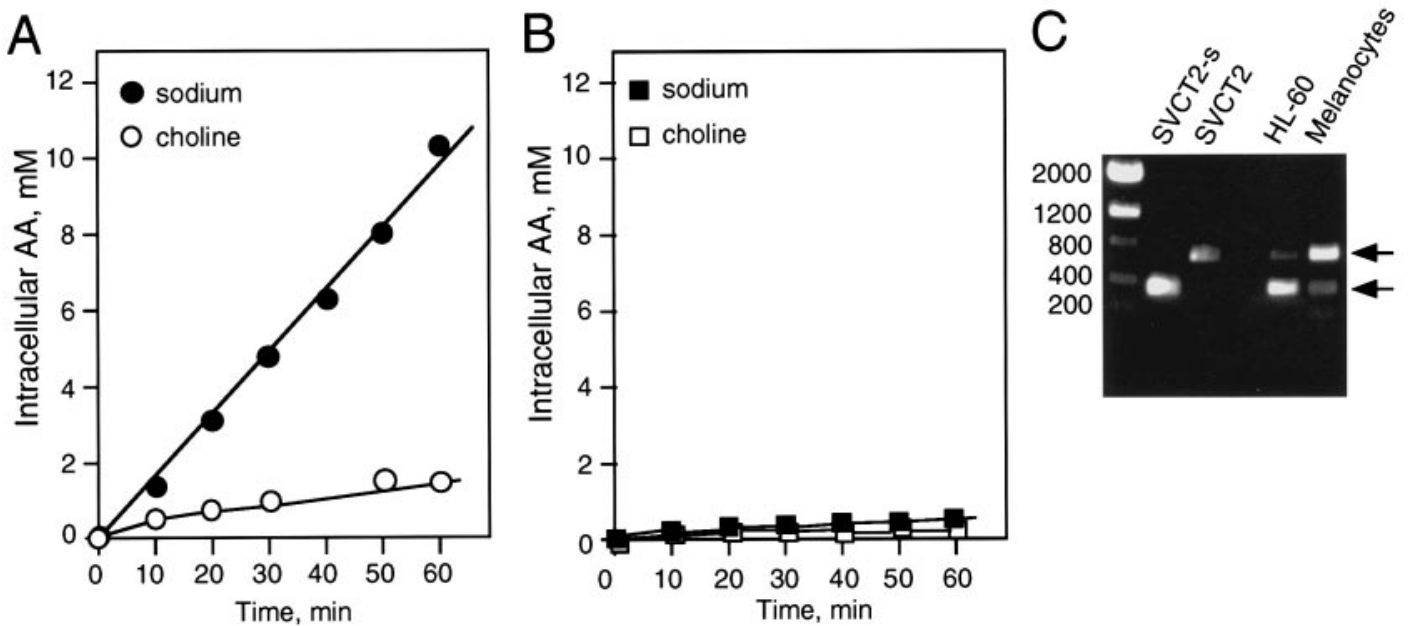

FIG. 5. Correlation between levels of short isoform of hSVCT2 and ability to transport ascorbate. (A) Melanocytes were incubated with 100 $\mu \mathrm{M}\left[{ }^{14} \mathrm{C}\right] \mathrm{AA}$ with and without sodium in the buffer. The cells were washed in cold PBS buffer, and the remaining radioactivity was counted and expressed as millimolar AA per million cells. (B) HL-60 cells were incubated with $100 \mu \mathrm{M}\left[{ }^{14} \mathrm{C}\right] \mathrm{AA}$ with and without sodium in the buffer. The cells were washed in cold PBS buffer, and the remaining radioactivity was counted and expressed as millimolar AA per million cells. (C) PCR was performed on cDNAs isolated from melanocytes and HL-60 cells using AAT2-300 and AAT2-R900 primers. The products were separated on a $1 \%$ agarose gel and visualized by ethidium bromide staining. 
human melanocytes that do (21) (Fig. 5A). As shown in Fig. 5A, melanocytes transport AA efficiently and in a sodiumdependent manner. We performed PCR using AAT2-300 and AAT2-R900 primers on cDNAs isolated from melanocytes and HL60 to determine the ratio between full-length hSVCT2 and its short isoform in these cell lines. Melanocytes showed a predominance of full-length hSVCT2, whereas HL-60 cells expressed predominantly its short isoform (Fig. 5C). This result correlates with the behavior of AA transport in these cells. The short isoform of hSVCT2 can inhibit AA transport in the HL-60 cell line and may, in part, be responsible for its inability to transport AA. Melanocytes, on the other hand, may not express sufficient amounts of the short isoform of hSVCT2 to impair direct transport of AA.

\section{DISCUSSION}

Human cells can take up vitamin $\mathrm{C}$ by two mechanisms: through the facilitative GLUTs as DHA or via the $\mathrm{Na}^{+}$-dependent cotransporters hSVCT1 and -2 as AA (10). These forms of transport are quite distinct, as the GLUTs do not require energy to facilitate transport down a concentration gradient and only transport vitamin $\mathrm{C}$ in the oxidized form as DHA. In contrast, the $\mathrm{Na}^{+}$-ascorbate cotransporters transport against a concentration gradient and require $\mathrm{Na}-\mathrm{K}$ ion exchange. We discovered a short isoform of hSVCT2 in fetal human brain tissue and molecularly characterized it. The short isoform is generated by alternative splicing and gives rise to a protein unable to transport AA. The short protein is missing transmembrane domains 5 and 6 and has a partially deleted domain 4. In transfected cells, the hSVCT2-short protein reaches the cell membrane, where it can be identified by immunostaining. Coexpression of hSVCT2-short with hSVCT1 and -2 indicated that the short isoform inhibited AA transport by both transporters. When coexpressed in 293T cells, hSVCT2 and hSVCT2-short were identified on the cell surface by immunostaining. Cell surface localization and functional inhibition of both hSVCT1 and -2 suggested that the dominantnegative effect comes about by protein-protein interactions; however, other mechanisms are possible, including effects on the trafficking of hSVCT2 between intracellular compartments and the cell membrane. Although alternatively spliced transporter proteins are well known (5), dominant-negative effects among transporters are uncommon. It was reported that two alternatively spliced isoforms of the $\mathrm{Na}^{+}$-dependent phosphate cotransporter $\mathrm{NaPi}-2, \mathrm{NaPi}-2 \alpha$ and $\mathrm{NaPi}-2 \gamma$, markedly inhibited NaPi-2 activity in Xenopus oocytes (22).

Spliced isoforms of transporters are common, and they are generally functional, resulting in a variety of biological consequences, including changes in kinetic properties, tissue distribution, or intracellular localization (for a review, see reference 5). For example, the GLUT-11 gene produces two proteins via alternative splicing - the long form, GLUT11-L, and the short form, GLUT11-S (30). The GLUT11-L cDNA uses all 13 exons of the gene to produce a protein containing 503 amino acids. The short form of GLUT11 cDNA is missing exon 2 and produces a 496 -amino-acid protein with a 14 -amino-acid $\mathrm{N}$ terminal difference from the long form. The two spliced forms are expressed at different levels in various tissues. GLUT11-S is expressed in most tissues except the liver, whereas
GLUT11-L is selectively expressed in the liver, brain, lung, and trachea (30). Both the long and short isoforms of the GLUT11 transporter are functional $(4,30)$.

The discovery of the nonfunctional hSVCT2-short isoform and its role as an inhibitor of AA transport suggests a partial explanation for an apparent paradox. While hSVCT2 is widely distributed in tissues, as assessed by mRNA expression, few cells can directly take up AA. Many established human cells lines do not transport AA $(1,8,16,26)$, similar to primary blood cells, such as neutrophils (27) and monocytes (16). Epithelial (HeLa) cells (2) and other tumor cell lines do not readily take up AA (1). The inhibitory function of hSVCT2short suggests that regulation of AA transport may in part relate to the balance of hSVCT2 and its short isoform. For example, HL-60 cells do not transport AA but express hSVCT2 mRNA. We found that in HL-60 cells there is a predominance of the hSVCT2-short isoform. On the other hand, human melanocytes that readily transport AA (21) had a predominance of the functional hSVCT2. In other cell lines unable to transport AA tested by PCR for hSVCT2-short mRNA expression, we found no correlation between the amount of hSVCT2-short mRNA and the ability of the cells to transport AA. We suspect that the major determinant of whether a cell can transport AA is the ability of hSVCT2 to reach the cell membrane. Little is known about the role of hSVCT2 in regulating transport of AA between intracellular compartments.

Inhibition of hSVCT1 required a substantial excess of hSVCT2-short, and inhibition of AA transport was seen only at a 10-fold excess of hSVCT2-short over hSVCT1. On the other hand, the inhibitory effect of hSVCT2-short on AA transport in cells expressing full-length hSVCT2 could be seen with as little as one molecule of hSVCT2-short per three molecules of full-length hSVCT2. This result indirectly suggests that protein-protein complex formation between the functional transporter and hSVCT2-short is responsible for the inhibitory effect of AA transport. The greater homology within the hSVCT2 and hSVCT2-short pair than between hSVCT1 and hSVCT2-short may explain the substantial quantitative difference between the dominant-negative effect of hSVCT2-short on full-length hSVCT2 and that on hSVCT1. The expression of hSVCT2-short had no effect on DHA transport by the facilitative GLUTs, indicating that it is not affecting membrane integrity.

The evolving picture is one of complex regulation of cellular vitamin $\mathrm{C}$ uptake and transmembrane compartmentalization. Relatively little is known about the regulation of alternative splicing, and it is not immediately apparent why direct cellular AA transport is so highly restricted. DHA uptake is regulated by the number and functionality of GLUTs, as well as the redox state (14), and may be enhanced by hormonal stimulation $(6,17,27)$. Perhaps AA transport is restricted to limit $\mathrm{Na}-\mathrm{K}$ exchange. Our results define a mechanism of AA uptake regulation that is dependent on the expression of a dominantnegative hSVCT2-short isoform.

The wide distribution of hSVCT2 and short-isoform mRNAs makes it possible that the regulation of ascorbate transport via protein-protein interaction between the two isoforms is an important mechanism of cellular vitamin $\mathrm{C}$ transport regulation. A recent report by Liang et al. (11) suggests 
that there is yet another mechanism of the regulation of vitamin $\mathrm{C}$ transport that occurs through the protein kinase $\mathrm{C}$ cascade. Protein kinase $\mathrm{C}$ activators caused a time- and concentration-dependent reduction of AA transport in COS-1 cells expressing AA transporters 1 and 2 (hSVCT1 and hSVCT2). For hSVCT1, the decrease in AA transport correlated with a redistribution of the transporter from the cell surface to intracellular membranes; however, for hSVCT2 there was no apparent change in transporter distribution. Kinetically, the reduction in hSVCT2 activity arose from a decrease in $V_{\max }$ with no change in affinity $\left(K_{m}\right)$. This result suggested that protein kinase $\mathrm{C}$-dependent reduction of AA transport mediated by hSVCT2 was due to reduced catalytic efficiency of the transporter.

Our findings identify a new hSVCT2 isoform that gives rise to a nonfunctional transmembrane protein capable of inhibiting AA transport through hSVCT2 in a dominant-negative manner via protein-protein interaction. The hSVCT2-short isoform also inhibits hSVCT1, although only if present in greater excess. These results define a new mechanism regulating the cellular accumulation of vitamin $\mathrm{C}$.

\section{ACKNOWLEDGMENTS}

We thank Matthias A. Hediger, Harvard Medical School, Boston, Mass., for providing the hSVCT1 cDNA and Oriana Borquez-Ojeda for experimental support with AA uptake in melanocytes and HL-60.

This work was supported by grants from the National Institutes of Health (CA 30388), the New York State Department of Health, and the Lebensfeld Foundation.

\section{REFERENCES}

1. Agus, D. B., J. C. Vera, and D. W. Golde. 1999. Stromal cell oxidation: a mechanism by which tumors obtain vitamin C. Cancer Res. 59:4555-4558.

2. Carcamo, J. M., A. Pedraza, O. Borquez-Ojeda, and D. W. Golde. 2002 Vitamin C suppresses TNF alpha-induced NF kappa B activation by inhibiting I kappa B alpha phosphorylation. Biochemistry 41:12995-13002.

3. Daruwala, R., J. Song, W. S. Koh, S. C. Rumsey, and M. Levine. 1999. Cloning and functional characterization of the human sodium-dependent vitamin C transporters hSVCT1 and hSVCT2. FEBS Lett. 460:480-484.

4. Doege, H., A. Bocianski, A. Scheepers, H. Axer, J. Eckel, H. G. Joost, and A. Schurmann. 2001. Characterization of human glucose transporter (GLUT) 11 (encoded by SLC2A11), a novel sugar-transport facilitator specifically expressed in heart and skeletal muscle. Biochem. J. 359:443-449.

5. Gamba, G. 2001. Alternative splicing and diversity of renal transporters. Am. J. Physiol Renal Physiol. 281:F781-F794.

6. Kodaman, P. H., and H. R. Behrman. 1999. Hormone-regulated and glucose-sensitive transport of dehydroascorbic acid in immature rat granulosa cells. Endocrinology 140:3659-3665.

7. Kyte, J., and R. F. Doolittle. 1982. A simple method for displaying the hydropathic character of a protein. J. Mol. Biol. 157:105-132.

8. Laggner, H., V. Besau, and H. Goldenberg. 1999. Preferential uptake and accumulation of oxidized vitamin C by THP-1 monocytic cells. Eur. J. Biochem. 262:659-665.

9. Levine, M., S. C. Rumsey, R. Daruwala, J. B. Park, and Y. Wang. 1999. Criteria and recommendations for vitamin C intake. JAMA 281:1415-1423.

10. Liang, W. J., D. Johnson, and S. M. Jarvis. 2001. Vitamin C transport systems of mammalian cells. Mol. Membr. Biol. 18:87-95.
11. Liang, W. J., D. Johnson, L. S. Ma, S. M. Jarvis, and L. Wei-Jun. 2002. Regulation of the human vitamin C transporters expressed in COS-1 cells by protein kinase C. Am. J. Physiol Cell Physiol. 283:C1696-C1704.

12. Lutsenko, E. A., J. M. Carcamo, and D. W. Golde. 2002. Vitamin C prevents DNA mutation induced by oxidative stress. J. Biol. Chem. 277:16895-16899.

13. Nishikimi, M., R. Fukuyama, S. Minoshima, N. Shimizu, and K. Yagi. 1994 Cloning and chromosomal mapping of the human nonfunctional gene for L-gulono-gamma-lactone oxidase, the enzyme for L-ascorbic acid biosynthesis missing in man. J. Biol. Chem. 269:13685-13688.

14. Nualart, F. J., C. I. Rivas, V. P. Montecinos, A. S. Godoy, V. H. Guaiquil, D. W. Golde, and J. C. Vera. 2003. Recycling of vitamin C by a bystander effect. J. Biol. Chem. 278:10128-10133.

15. Pear, W. S., G. P. Nolan, M. L. Scott, and D. Baltimore. 1993. Production of high-titer helper-free retroviruses by transient transfection. Proc. Natl. Acad. Sci. USA 90:8392-8396.

16. Perez-Cruz, I., J. M. Carcamo, and D. W. Golde. 2003. Vitamin C inhibits FAS-induced apoptosis in monocytes and U937 cells. Blood 102:336-343.

17. Qutob, S., S. J. Dixon, and J. X. Wilson. 1998. Insulin stimulates vitamin C recycling and ascorbate accumulation in osteoblastic cells. Endocrinology 139:51-56.

18. Rajan, D. P., W. Huang, B. Dutta, L. D. Devoe, F. H. Leibach, V. Ganapathy, and P. D. Prasad. 1999. Human placental sodium-dependent vitamin C transporter (SVCT2): molecular cloning and transport function. Biochem. Biophys. Res. Commun. 262:762-768.

19. Rumsey, S. C., R. Daruwala, H. Al-Hasani, M. J. Zarnowski, I. A. Simpson, and M. Levine. 2000. Dehydroascorbic acid transport by GLUT4 in Xenopus oocytes and isolated rat adipocytes. J. Biol. Chem. 275:28246-28253.

20. Sotiriou, S., S. Gispert, J. Cheng, Y. Wang, A. Chen, S. Hoogstraten-Miller, G. F. Miller, O. Kwon, M. Levine, S. H. Guttentag, and R. L. Nussbaum. 2002. Ascorbic-acid transporter Slc23a1 is essential for vitamin C transport into the brain and for perinatal survival. Nat. Med. 8:514-517.

21. Spielholz, C., D. W. Golde, A. N. Houghton, F. Nualart, and J. C. Vera. 1997. Increased facilitated transport of dehydroascorbic acid without changes in sodium-dependent ascorbate transport in human melanoma cells. Cancer Res. 57:2529-2537.

22. Tatsumi, S., K. Miyamoto, T. Kouda, K. Motonaga, K. Katai, I. Ohkido, K. Morita, H. Segawa, Y. Tani, H. Yamamoto, Y. Taketani, and E. Takeda. 1998. Identification of three isoforms for the $\mathrm{Na}+$-dependent phosphate cotransporter (NaPi-2) in rat kidney. J. Biol. Chem. 273:28568-28575.

23. Tsukaguchi, H., T. Tokui, B. Mackenzie, U. V. Berger, X. Z. Chen, Y. Wang, R. F. Brubaker, and M. A. Hediger. 1999. A family of mammalian $\mathrm{Na}+-$ dependent L-ascorbic acid transporters. Nature 399:70-75.

24. Vera, J. C., C. I. Rivas, J. Fischbarg, and D. W. Golde. 1993. Mammalian facilitative hexose transporters mediate the transport of dehydroascorbic acid. Nature 364:79-82.

25. Vera, J. C., C. I. Rivas, F. V. Velasquez, R. H. Zhang, I. I. Concha, and D. W. Golde. 1995. Resolution of the facilitated transport of dehydroascorbic acid from its intracellular accumulation as ascorbic acid. J. Biol. Chem. 270: 23706-23712.

26. Vera, J. C., C. I. Rivas, R. H. Zhang, C. M. Farber, and D. W. Golde. 1994. Human HL-60 myeloid leukemia cells transport dehydroascorbic acid via the glucose transporters and accumulate reduced ascorbic acid. Blood 84:16281634.

27. Vera, J. C., C. I. Rivas, R. H. Zhang, and D. W. Golde. 1998. Colonystimulating factors signal for increased transport of vitamin $\mathrm{C}$ in human host defense cells. Blood 91:2536-2546.

28. Wang, H., B. Dutta, W. Huang, L. D. Devoe, F. H. Leibach, V. Ganapathy, and P. D. Prasad. 1999. Human $\mathrm{Na}^{+}$-dependent vitamin $\mathrm{C}$ transporter 1 (hSVCT1): primary structure, functional characteristics and evidence for a non-functional splice variant. Biochim. Biophys. Acta 1461:1-9.

29. Wang, Y., B. Mackenzie, H. Tsukaguchi, S. Weremowicz, C. C. Morton, and M. A. Hediger. 2000. Human vitamin C (L-ascorbic acid) transporter SVCT1. Biochem. Biophys. Res. Commun. 267:488-494.

30. Wu, X., W. Li, V. Sharma, A. Godzik, and H. H. Freeze. 2002. Cloning and characterization of glucose transporter 11, a novel sugar transporter that is alternatively spliced in various tissues. Mol. Genet. Metab. 76:37-45. 